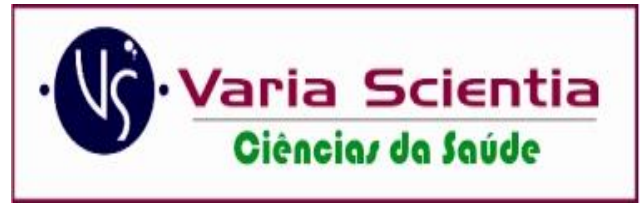

e-ISSN 2446-8118

\title{
CARACTERIZAÇÃO DAS TENTATIVAS DE SUICÍDIO POR EXPOSIÇÃO A AGENTES TÓXICOS
}

\section{CHARACTERIZATION OF SUICIDE ATTEMPTS BY EXPOSURE TO TOXIC AGENTS CARACTERIZACIÓN DE INTENTOS DE SUICIDIO POR EXPOSICIÓN A AGENTES TÓXICOS}

\author{
Everlaine Silva dos Santos ${ }^{1}$ \\ Maristela Salete Maraschin ${ }^{2}$ \\ Leda Aparecida Vanelli Nabuco de Gouvea ${ }^{3}$ \\ Gicelle Galvan Machineski ${ }^{4}$
}

\begin{abstract}
RESUMO: Objetivo: caracterizar o perfil dos indivíduos que tentaram suicídios por exposição a agentes tóxicos, registrados em um Centro de Informação e Atendimento Toxicológico, no período de 01 de setembro de 2018 a 02 de fevereiro de 2020. Materiais e métodos: estudo descritivo, retrospectivo e documental, com análise quantitativa. Este estudo teve, como fontes secundárias de dados, as fichas de atendimento, utilizadas pelo Centro de Informação e Atendimento Toxicológico, no período de 01 de setembro de 2018 a 02 de fevereiro de 2020, totalizando 102 fichas de intoxicação. A coleta de dados ocorreu nos meses de março e abril de 2020. O instrumento de coleta de dados teve 11 variáveis, pertinentes ao indivíduo, quanto à exposição e à evolução clínica do indivíduo. Resultados: Do total da amostra, a maior ocorrência foi com o gênero feminino; a idade predominante foi menor que 20 anos e prevaleceu a baixa escolaridade. Os medicamentos e os agrotóxicos foram as substâncias mais encontradas. Conclusão: Os resultados apresentam as intoxicações exógenas como um sério problema de saúde pública, que exige elaboração de ações e políticas no âmbito da gestão em saúde, de modo a reduzir os fatores de risco e melhorar a qualidade de vida.
\end{abstract}

DESCRITORES: Suicídio; Tentativa de suicídio; Intoxicação.

ABTRACT: Objective: characterize the profile of individuals who try to commit suicide by exposure to toxic agents, registered in a Toxicological Information and Service Center, between September $1^{\text {st }}$, 2018 and February $2^{\text {nd }}, 2000$. Method: descriptive, retrospective, and documental study, with quantitative analysis. This study had, as secondary source of data, the attendance files, used by Toxicological Information and Service Center, from September $01^{\text {st }} 2018$ to February $02^{\text {nd }} 2020$, totalizing 103 intoxication files. The instrument of data collection had 11 variables, pertinent to the individual, related to the exposition and clinical evolution of the individual. Results: From the total

\footnotetext{
${ }^{1}$ Universidade Estadual do Oeste do Paraná (UNIOESTE) - Brasil. Acadêmica do 5o Ano do Curso de Enfermagem da Universidade Estadual do Oeste do Paraná (UNIOESTE), campus de Cascavel - PR.

${ }^{2}$ Universidade Estadual do Oeste do Paraná (UNIOESTE). Enfermeira, mestre. Docente da Universidade Estadual do Oeste do Paraná (UNIOESTE), campus Cascavel - PR.

${ }^{3}$ Universidade Estadual do Oeste do Paraná (UNIOESTE). Enfermeira, mestre. Docente da Universidade Estadual do Oeste do Paraná (UNIOESTE), campus Cascavel - PR.

${ }^{4}$ Universidade Estadual do Oeste do Paraná (UNIOESTE). Doutora, enfermeira, docente da Universidade Estadual do Oeste do Paraná (UNIOESTE), campus de Cascavel - PR.
} 
of the data, the highest occurrence was the feminine genre; the predominant age was younger than 20 years old and predominated the low education level. Medication and pesticides were the most found substances. Conclusion: The results present the exogenous intoxication as a serious public health problem, which requires the elaboration of actions and polices on the scope of healthcare management, in order to reduce the risk factors and to improve the quality of life.

DESCRIPTORS: Suicide; Suicide attempt; Intoxication.

RESUMEN: Objetivo: caracterizar el perfil de individuos que intentaron suicidarse por exposición a agentes tóxicos, registrados en un Centro de Información y Atención Toxicológica, en el período de 01 de septiembre de 2018 al 02 de febrero de 2020. Método: estudio descriptivo, retrospectivo y documental, con análisis cuantitativo. Este estudio tuvo como fuentes secundarias de datos los formularios de asistencia, utilizados por el Centro de Información y Atención Toxicológica, del 1 de septiembre de 2018 al 2 de febrero de 2020, totalizando 102 formularios de intoxicación. El instrumento de recolección de datos contó con 11 variables, relevantes para el individuo, en cuanto a exposición y evolución clínica del individuo. Resultados: Del total de la muestra, la mayor ocurrencia fue con el género femenino; la edad predominante fue menor de 20 años y predominó la baja escolaridad. Los medicamentos y pesticidas fueron las sustancias más comúnmente encontradas. Conclusión: Los resultados presentan las intoxicaciones exógenas como un grave problema de salud pública, que requiere la elaboración de acciones y políticas en el ámbito de la gestión sanitaria, con el fin de reducir los factores de riesgo y mejorar la calidad de vida.

DESCRIPTORES: Suicidio; Intento de suicidio; Intoxicación.

\section{INTRODUÇÃO}

O suicídio pode ser conceituado como um ato intencional deliberado e levado a cabo por alguém que tem consciência do resultado final. Por sua vez, quando a pessoa o suicida não consegue êxito, passa a ser classificado pela literatura como tentativa de suicídio $^{1}$. O suicídio é um fenômeno social que constitui um grave problema de saúde pública ${ }^{2}$. É resultado de uma complexa interação de fatores biológicos, genéticos, psicológicos, sociológicos, culturais e ambientais ${ }^{1}$.

De acordo com a literatura, uma pessoa comete suicídio, a cada 45 segundos, o que figura entre as três principais causas de morte na população economicamente ativa. Dessa forma, o suicídio é responsável por um milhão de óbitos, anualmente; o Brasil, por ser um país populoso, possui um coeficiente de mortalidade considerado relativamente baixo, mas, mesmo assim, está entre os dez países que registram maiores números absolutos de suicídio $^{3}$. A maioria dos suicídios no mundo está relacionada a doenças psiquiátricas. Entre esses, depressão, uso de substâncias lícitas e psicose constituem os fatores de risco mais relevantes, mas também ansiedade, transtornos relacionados à personalidade, alimentação e trauma, além de transtornos mentais orgânicos, que contribuem significativamente para causas de morte não naturais em comparação com a população em geral ${ }^{4}$.

No Brasil, o suicídio, principalmente entre jovens de 15 a 29 anos, figura como a quarta maior causa de morte ${ }^{2}$. Conforme dados do Departamento de Informática do Sistema Único de Saúde, do Ministério da Saúde/DATASUS/MS, a taxa de óbitos por suicídio foi de 6,13 por 100 mil habitantes, em 2016 (9,8 para homens e 2,5 para mulheres), o que representou 11.433 mortes $^{2}$.

As taxas de suicídios efetivados são mais comuns nos homens do que em mulheres, diferentemente das tentativas de suicídio, que são mais comuns nas mulheres do que nos homens. De um modo geral, fica evidente que essas taxas variam ao longo da vida e entre os gêneros. Nesse sentido, os métodos mais utilizados são influenciados pela cultura e pelo acesso que se tem a eles ${ }^{4}$.

São aproximadamente dezesseis milhões de tentativas de suicídio por ano, ou seja, uma tentativa a cada dois segundos. No Brasil e na maioria dos países do mundo, há uma carência de informações em relação a 
esses dados, pois há baixa qualidade e o problema das subnotificações, que são reconhecidos pela própria Organização Mundial da Saúde, principalmente, nos países onde o comportamento suicida é considerado crime $^{5}$. Diante disso, evidencia-se um comprometimento em relação ao resultado geral.

Mesmo que a situação seja preocupante, é possível ser prevenida; o tabu em relação ao tema do suicídio impossibilita a busca por ajuda, que pode evitar mortes. Da mesma maneira, considera-se que abordar, de forma responsável, o assunto pode representar um fator de prevenção e ajudar a diminuir os riscos em relação a tal realidade, podendo, inclusive, contrapor-se a suas causas ${ }^{6}$. Diante disso, a compreensão e o estudo do comportamento suicida tornam-se uma necessidade premente, visto que as tentativas de suicídio acarretam custo considerável ao sistema de saúde e à sociedade ${ }^{7}$.

As opções de cuidados disponíveis e os seus benefícios devem ser amplamente divulgados, de tal forma que as respostas da população, em geral, dos profissionais, dos meios de comunicação, dos formuladores de políticas e dos políticos sejam o reflexo dos melhores conhecimentos disponíveis ${ }^{8}$. Uma tentativa de suicídio é o principal fator de risco para sua futura concretização, após uma tentativa, estima-se que o risco de suicídio aumente em pelo menos cem vezes em relação aos índices presentes na população geral ${ }^{3}$. Por isso, essas tentativas devem ser encaradas com seriedade, como um sinal de alerta a indicar a atuação de fenômenos psicossociais complexos. É essencial oferecer especial atenção a uma pessoa que tentou se suicidar, visto que se configura como uma das principais estratégias para se evitar um futuro suicídio ${ }^{3}$.

Diante do exposto, questionou-se: como acontecem as tentativas de suicídios e quem são os indivíduos que tentaram suicídio? Para tanto, este estudo tem como objetivo caracterizar o perfil dos indivíduos que tentaram suicídios por exposição a agentes tóxicos, registrados em um Centro de Informação e Atendimento Toxicológico, no período de 01 de setembro de 2018 a 02 de fevereiro de 2020.
Identificar o perfil dessas pessoas é elemento essencial de quaisquer estratégias abrangentes de prevenção do suicídio, pois elas estão em alto risco para um futuro suicídio; sendo assim, a melhor forma de evitar o ato, em si, é a providência de cuidados e acompanhamento.

\section{MATERIAIS E MÉTODOS}

Este estudo é caracterizado como descritivo, retrospectivo e documental, com análise quantitativa dos casos de tentativa de suicídio por exposição a agentes tóxicos registrados pelo Centro de Informação e Assistência Toxicológica (CIATOX), localizado no município de Cascavel/PR. O serviço é responsável por atender qualquer município do Estado do Paraná que solicite informações. O Ciatox funciona no Hospital Universitário do Oeste do Paraná (HUOP), desde o ano de 2005, com atendimento de plantão permanente, em turnos de 24 horas, sete dias por semana, integrado aos demais serviços do hospital, principalmente, ao pronto-socorro e ao laboratório de análises clínicas.

O Ciatox fornece informações presenciais dos indivíduos expostos a agentes tóxicos e/ou intoxicados, para a equipe médica e de enfermagem do HUOP, como também informações por telefone aos profissionais de outras unidades de saúde da cidade de Cascavel e de outros municípios. Suas atividades são desenvolvidas por uma equipe de profissionais da saúde composta de médicos e farmacêuticos, além de contar com o auxílio de estagiários (estudantes de graduação dos cursos de Enfermagem, Medicina e Farmácia).

Este estudo teve, como fonte secundária de dados, as fichas de atendimento (DATATOX), utilizadas pelo Ciatox, no período de 01 de setembro de 2018 a 02 de fevereiro de 2020, totalizando um período de 18 meses. A escolha desse período efetivou-se a partir do momento em que o Ciatox começou a atender em plantão permanente, em turnos de 24 horas. Em momento anterior, o serviço atendia em um período reduzido de oito horas diárias. 
As fichas utilizadas no Ciatox, para registro de atendimento, são um instrumento formal, considerado pela Associação Brasileira de Centros de Informação e Assistência Toxicológica (ABRACIT). Essas fichas possuem informações pertinentes ao indivíduo, as circunstâncias da ocorrência e a evolução clínica. Para a coleta de dados, foi construído um instrumento, que consiste em um roteiro, o qual contém três partes; a primeira possui as variáveis pertinentes ao indivíduo exposto, tais como: gênero, idade, escolaridade, agente tóxico, área de residência, raça, ocupação/profissão e município de residência; a segunda parte evidencia dados quanto à exposição: local da exposição, via da exposição; já a terceira parte está relacionada à evolução clínica do paciente, a saber: se teve um desfecho assintomático, se evoluiu para cura, se teve sequela, se teve algum diagnóstico diferencial, ignorado, ou se evoluiu para óbito, totalizando 11 variáveis.

No período do estudo, ocorreram 102 casos. Foram excluídos os casos, nos quais as fichas de atendimento estavam fora do período do estudo.

Os dados coletados foram tabulados em planilhas no Excel, por meio da estatística descritiva simples, com apresentação de números inteiros e percentuais, calculando a frequência relativa e absoluta. Dessa forma, permite-se que os dados levantados sejam organizados e interpretados.

Este estudo faz parte de um projeto de pesquisa mais amplo, que incluiu a caracterização do perfil das doenças e agravos de notificações obrigatórias, bem como das infecções relacionadas com a assistência em saúde, o qual foi aprovado pelo Comitê de Ética em Pesquisa, que Envolve Seres Humanos, conforme parecer número 4.047.913/2020 e CAAE 90600318.3.0000.0107, da Universidade Estadual do Oeste do Paraná/UNIOESTE, respeitando as diretrizes da Resolução 466 de $2012^{9}$.

\section{RESULTADOS}

Artigo Original

No período estudado, foram registrados $102(100 \%)$ casos de tentativa de suicídio por exposição a agentes tóxicos, no Ciatox, os quais correspondem a uma parcela do total de casos ocorridos na região, pois nem todos chegam ao serviço.

A Tabela I mostra as variáveis quanto ao sexo, faixa etária, escolaridade, agente tóxico e área de residência. De acordo com o gênero, houve predomínio do feminino, visto que $57(55,8 \%)$ eram mulheres e $45(44,1 \%)$ homens, ou seja, a população feminina, que tentou suicídio, foi $11,7 \%$ maior do que a população masculina, totalizando 12 tentativas registradas a mais.

A distribuição por faixa etária apresentou, entre os jovens, menores de 20 anos de idade, $32(31,3 \%)$ dos casos, seguido de 25 a 29 anos, que totalizaram $20(19,6 \%)$; os de 20 a 24 anos são $13(12,7 \%)$ dos casos. A faixa etária com menor incidência foi de 50 a 54 anos de idade, com 3 (2,9\%) casos, seguido da faixa dos 30 aos 34 (3,9\%), com quatro casos.

Em relação à escolaridade, o ensino fundamental incompleto e os casos ignorados/brancos, ambos tiveram o mesmo percentual, de 38 casos $(37,2 \%)$, seguidos pelo ensino médio incompleto, $16(15,6 \%)$, e do ensino médio completo $6(5,8 \%)$.

Os agentes tóxicos mais usados nas tentativas de suicídio foram os medicamentos, $55(53,9 \%)$, seguidos dos agrotóxicos, 16 $(15,6 \%)$; na sequência, houve o uso simultâneo de dois ou mais agentes tóxicos, pertencentes a diferentes grupos, com 13 (12,7\%) dos casos. No que se refere à área de residência, os casos ocorreram com maior frequência em área urbana, 86 (84,6\%); já em área rural, foram 16 $(15,6 \%)$ do total dos casos.

$\mathrm{Na}$ Tabela 1, descrevem-se as variáveis quanto ao gênero, faixa etária, escolaridade, agente toxicológico e área de residência dos indivíduos que tentaram suicídios por exposição a agentes tóxicos. 
TABELA 1 - Variáveis quanto ao sexo, faixa etária, escolaridade, agente toxicológico e área de residência dos indivíduos que tentaram suicídios por exposição a agentes. Cascavel/PR, 2020.

\begin{tabular}{|c|c|c|c|c|c|c|c|c|}
\hline \multirow{3}{*}{ VARIÁVEL } & \multicolumn{8}{|c|}{ ANO DE OCORRÊNCIA } \\
\hline & \multicolumn{2}{|c|}{$\mathbf{2 0 1 8}^{1}$} & \multicolumn{2}{|c|}{$2019^{2}$} & \multicolumn{2}{|c|}{$2020^{3}$} & \multicolumn{2}{|c|}{ TOTAL } \\
\hline & $\mathbf{N}$ & $\%$ & $\mathbf{N}$ & $\%$ & $\mathbf{N}$ & $\%$ & $\mathbf{N}$ & $\%$ \\
\hline \multicolumn{9}{|l|}{ Sexo } \\
\hline Feminino & 6 & 66,6 & 43 & 55,1 & 8 & 53,3 & 57 & 55,8 \\
\hline Masculino & 3 & 33,3 & 35 & 44,8 & 7 & 46,6 & 45 & 44,1 \\
\hline \multicolumn{9}{|l|}{ Faixa etária } \\
\hline$<20$ Anos & 4 & 44,1 & 24 & 30,7 & 4 & 26,6 & 32 & 31,3 \\
\hline $25-29$ & 2 & 22,2 & 15 & 19,2 & 3 & 20 & 20 & 19,6 \\
\hline $20-24$ & 1 & 11,1 & 11 & 14,1 & 1 & 6,6 & 13 & 12,7 \\
\hline $35-39$ & 0 & & 8 & 10,2 & 3 & 20 & 11 & 10,7 \\
\hline $40-44$ & 1 & 11,1 & 6 & 7,6 & 1 & 6,6 & 8 & 7,8 \\
\hline $45-49$ & 0 & & 5 & 6,4 & 1 & 6,6 & 6 & 5,8 \\
\hline $55<$ & 0 & & 5 & 6,4 & 0 & & 5 & 4,9 \\
\hline $30-34$ & 1 & 11,1 & 3 & 3,8 & 0 & & 4 & 3,9 \\
\hline $50-54$ & 0 & & 2 & 2,5 & 1 & 6,6 & 3 & 2,9 \\
\hline \multicolumn{9}{|l|}{ Escolaridade } \\
\hline Ensino fundamental incompleto & 3 & 33,3 & 27 & 34,6 & 8 & 53,3 & 38 & 37,2 \\
\hline Ignorado/branco & 3 & 33,3 & 32 & 40 & 3 & 20 & 38 & 37,2 \\
\hline Ensino médio incompleto & 3 & 33,3 & 9 & 11,5 & 4 & 26,6 & 16 & 15,6 \\
\hline Ensino médio completo & 0 & & 6 & 7,6 & 0 & & 6 & 5,8 \\
\hline Ensino fundamental completo & 0 & & 3 & 3,8 & 0 & & 3 & 2,9 \\
\hline Ensino superior incompleto & 0 & & 1 & 1,2 & 0 & & 1 & 0,9 \\
\hline Ensino superior completo & 0 & & 0 & & 0 & & 0 & \\
\hline \multicolumn{9}{|l|}{ Agente tóxico } \\
\hline Medicamento & 4 & 44,4 & 40 & 51,2 & 11 & 73,3 & 55 & 53,9 \\
\hline Agrotóxico & 2 & 22,2 & 13 & 16,6 & 1 & 6,6 & 16 & 15,6 \\
\hline $\begin{array}{l}\text { Associação de duas ou mais } \\
\text { substâncias }\end{array}$ & 2 & 22,2 & 11 & 14,1 & 0 & & 13 & 12,7 \\
\hline Raticida & 1 & 11,1 & 5 & 6,4 & 3 & 20 & 9 & 8,8 \\
\hline $\begin{array}{l}\text { Produto químico de uso } \\
\text { residencial/industrial }\end{array}$ & 0 & & 5 & 6,4 & 0 & & 5 & 4,9 \\
\hline Drogas de abuso & 0 & & 3 & 3,8 & 0 & & 3 & 2,9 \\
\hline Plantas e fungos & 0 & 22,2 & 1 & 1,2 & 0 & & 1 & 0,9 \\
\hline \multicolumn{9}{|l|}{ Área de residência } \\
\hline Urbana & 8 & 88,8 & 66 & 84,6 & 12 & 80 & 86 & 84,3 \\
\hline Rural & 1 & 11,1 & 12 & 15,3 & 3 & 20 & 16 & 15,6 \\
\hline
\end{tabular}

\footnotetext{
Fonte: Fichas de atendimentos Ciatox/2020

1 - Período de setembro, outubro, novembro e dezembro/2018

2 - Período de janeiro a dezembro/2019

3 - Período janeiro e fevereiro/2020
}

Para raça/cor, em ambos os sexos, prevaleceu a cor branca, com 50 casos (49\%); as situações, que não foram identificadas, totalizaram $32(31,3 \%)$, seguidas dos casos de pacientes de cor parda, com $17(16,6 \%)$, e negros, com $1(0,9 \%)$ dos casos. Quanto à profissão/ocupação, também ocorreu elevado percentual, com $31(30,3 \%)$ dos casos não identificados (não constava a profissão do indivíduo), seguidos dos estudantes, com 23 $(22,5 \%)$ dos casos, e do grupo dos desempregados, com 14 (13,7\%).

Ao analisar os registros, quanto ao município de residência, houve uma variação expressiva. O município de residência com mais registros foi Cascavel/PR, com 34 $(33,3 \%)$ dos casos; posteriormente, há o município de Coronel Vivida, com oito $(7,8 \%)$, e o município de Toledo, com seis $(5,8 \%)$ dos casos registrados.

Em relação ao local de exposição ao agente tóxico, a residência/moradia habitual aparece em maior proporção, em 98 (96\%) casos, com dois (1,9\%) ignorados e, igualmente, dois $(1,9 \%)$ casos ocorreram em ambiente externo. Dos 102 casos registrados de tentativa de suicídio, todos os indivíduos foram expostos ao agente tóxico por via oral. 
Artigo Original

Quanto à evolução clínica dos indivíduos, $79(77,4 \%)$ tiveram desfecho ignorado, $21(20,5 \%)$ ficaram assintomáticos e dois $(1,9 \%)$ faleceram. Dos dois indivíduos que morreram, um deles era do gênero masculino, na faixa etária de 35 a 39 anos, cuja escolaridade não foi identificada; o agente tóxico utilizado foi agrotóxico e era residente da área rural. O outro óbito aconteceu com uma pessoa do gênero feminino, menor que 20 anos, com ensino fundamental incompleto, cujo agente tóxico utilizado foi medicamento, a qual era residente da área urbana.

\section{DISCUSSÃO}

$\mathrm{O}$ presente estudo evidenciou que a maioria dos indivíduos, envolvidos nos casos de tentativa de suicídio, registrados pelo CIAtox/Cascavel, foram do sexo feminino. Um estudo, realizado no Estado de Pernambuco, com dados do Sistema de Informação dos Agravos de Notificação (SINAN), do Centro de Atendimento Toxicológico (CIAtox) e do Sistema de Informação de Mortalidade (SIM), quanto ao perfil das intoxicações, verificou que os casos acometem com mais frequência as pessoas do sexo feminino, no perfil de morbidade; já os homens, apresentaram maior letalidade ${ }^{10}$. De acordo com a literatura, as pessoas do sexo masculino são mais suscetíveis ao suicídio, tentam menos frequentemente, mas com métodos mais efetivos e, portanto, apresentam êxito no maior número de vezes, enquanto as mulheres

apesar de tentarem mais o suicídio, têm menos êxito, por visarem drogas menos tóxicas ${ }^{11}$.

Neste estudo, o maior número de casos de tentativas de suicídio apresentou-se em jovens menores de 20 anos de idade, totalizando $31,3 \%$ dos casos. A predominância do perfil etário, neste caso, é semelhante ao que foi encontrado em outros estudos. A ação de autodestruição é classificada como uma espécie de violência e que esta afeta principalmente o segmento populacional formado por adolescentes e adultos jovens ${ }^{12}$. Dessa forma, é possível compreender que, nessa faixa etária, exista maior fragilidade ao lidar com determinadas situações.
A freuência de comportamentos como um desafio para toda sociedade e para a saúde pública, onde tabus devem ser quebrados, a fim de que se notifique as tentativas para que colaborem com estudos epidemiológicos com finalidades de estabelecer estratégias de prevenção de casos novos e reincidências ${ }^{13}$.

Outro dado, encontrado no presente estudo e, também, consonante com outras pesquisas, foi o predomínio de casos com baixa escolaridade, seguido dos ignorados/brancos. O baixo nível escolar, especialmente o analfabetismo, apresenta-se como um fator de risco para a realização de violência autoprovocada por intoxicação exógena. A falta de escolarização está, geralmente, relacionada a dificuldades socioeconômicas, podendo resultar em grandes prejuízos à qualidade de vida individual e familiar e, consequentemente, aumentar o risco de comportamentos suicidas ${ }^{14}$.

Os agentes tóxicos envolvidos, com maior frequência, foram, primeiramente os medicamentos; na sequência, estão os agrotóxicos, seguidos da associação de duas ou mais substâncias. Um estudo, que se propôs a estimar a frequência do uso das substâncias tóxicas, como meio para tentativas e suicídio, indicou um perfil jovem, tanto nas tentativas quanto nos suicídios, principalmente, por medicamentos psicotrópicos e agrotóxicos ${ }^{15}$. Outro estudo, realizado em um serviço de urgência e emergência do município de Teresina, Piauí, em relação aos agentes tóxicos utilizados, revelou que: os medicamentos prevaleceram tanto nos casos não fatais quanto nos fatais ${ }^{14}$. Também: observou-se que, a utilização de agrotóxicos apresenta maior tendência de evoluir para um desfecho fatal quando comparado ao uso de medicamentos ${ }^{14}$. Contudo, nesta pesquisa, foi possível observar o percentual de tentativas de suicídio em residentes de áreas urbanas. Este dado pode estar associado às intensas mudanças da vida atual nos grandes centros urbanos, associadas ao estresse, depressão e desesperança, os quais vêm atingindo cada vez mais pessoas em todo o mundo ${ }^{11}$. Realizar a tentativa na área rural mostra-se significativamente associado ao autodestrutivos entre jovens apresenta-se 
suicídio, o que pode estar relacionado à maior dificuldade de acesso aos serviços de saúde ${ }^{14}$. Outro estudo reforça que apesar de sugerir subnotificação, dados mostram que pessoas ocupadas com a atividade na agricultura são mais comumente acometidas ${ }^{10}$.

$\mathrm{O}$ decorrer do estudo mostrou que, de acordo com a raça/cor, $49 \%$ dos indivíduos eram brancos, $16,6 \%$ pardos e $0,9 \%$ negros. Esses resultados vão ao encontro de dados de um boletim, que apresenta a situação epidemiológica das tentativas e óbitos por suicídio, no Brasil, vinculados à atuação da rede de atenção à saúde . $^{6}$

Em relação à profissão/ocupação, destaca-se o elevado percentual dos casos não identificados (30,3\%). Esses dados são congruentes com o descrito na literatura, assim como em outro estudo, no qual chama atenção quanto ao preenchimento inadequado, frequentemente incompleto, das fichas utilizadas pelo serviço para o registro desses eventos ${ }^{16}$. Por sua vez, neste estudo, percebeuse a necessidade do preenchimento adequado das fichas por parte dos profissionais, a fim de contribuir com maior precisão quanto aos dados relativos ao atendimento dos eventos toxicológicos registrados pelo serviço. Apesar das falhas, mais importante é a contribuição propiciada pelo serviço junto aos demais Centros de Informação e Assistência Toxicológica do país, o que confere visibilidade às intoxicações, hoje, consideradas como um importante problema de Saúde Pública ${ }^{16}$.

O percentual de tentativas de suicídio entre estudantes $(22,5 \%)$, encontrados neste estudo, efetivou-se pelo fato de que a maioria dos casos apresentaram idade menor que vinte anos, ou seja, a maioria se encontrava em atividade escolar.

Apesar da variação, a maioria dos casos atendidos no serviço pesquisado moravam no município de Cascavel, fato que pode ser explicado, em parte, pela localização do CIAtox/PR. Trata-se de um dos quatro centros especializados no atendimento de indivíduos expostos a agentes tóxicos e/ou intoxicados do estado do Paraná.

No Brasil, a própria casa é o cenário mais frequente de suicídios, seguida pelos hospitais $^{3}$. No presente estudo, quanto ao local de exposição ao agente tóxico, a maior parte dos indivíduos, que tentaram o ato, em si, tentaram na própria residência/moradia habitual; esse resultado é semelhante ao encontrado na literatura, onde o local de maior acesso aos agentes tóxicos é o próprio lar do indivíduo, de modo que as substâncias tóxicas usadas estão disponíveis para seu próprio uso ou de algum familiar ${ }^{12}$.

Quanto à via de exposição, a totalidade dos indivíduos utilizou a via oral para se autointoxicar (100\%), o que corrobora os resultados encontrados em outras pesquisas ${ }^{7}$, pertinente a evolução clínica dos indivíduos, $77,4 \%$ tiveram desfecho ignorado, 20,5\% assintomático e $1,9 \%$ faleceram. Dentre as dimensões da qualidade, os dados, preenchidos como ignorado/em branco, atingem a magnitude de agravos ou doenças ${ }^{15}$. Nesse período do estudo, ocorreram dois óbitos $(1,9 \%)$; quase a totalidade de casos de intoxicação obteve êxito na conduta da equipe multiprofissional, durante o atendimento de urgência/emergência, junto ao CIAtox. Contatar os centros de informação e controle de tóxicos para a classificação de intoxicações favorece a informação a respeito, além de promover o aconselhamento aos profissionais de saúde sobre o atendimento mais adequado, de maneira que serve de referência ao tratamento hospitalar, no caso de intoxicações mais severas ${ }^{17}$. Diante do exposto, revela-se a necessidade de trabalhar alguns aspectos importantes, por exemplo, oferecer continuidade de suporte aos casos de tentativas de suicídio, após o atendimento hospitalar. Uma tentativa de suicídio é o principal fator de risco para sua futura concretização ${ }^{3}$.

\section{CONCLUSÃO}

O desenvolvimento deste estudo possibilitou conhecer o perfil das tentativas de suicídio por intoxicação exógena, que chegaram até um atendimento de saúde e, posteriormente, a um Centro de Informação e Atendimento toxicológico. Ademais, proporcionou uma reflexão acerca da necessidade de desmitificar e diminuir as taxas alarmantes na população, destacando a necessidade de se discutir mais sobre o assunto, como também compreender a 
necessidade de propiciar continuidade à assistência após o atendimento hospitalar, a fim de evitar uma nova tentativa ou um futuro suicídio.

As taxas de tentativas de suicídio, encontradas neste estudo, entre adolescentes e os jovens adultos de baixa escolaridade, por intoxicação exógena, foram elevadas; o fácil acesso a determinados grupos de agentes tóxicos, utilizados pelas vítimas, deve favorecer discussões sobre a necessidade de medidas restritivas por parte dos órgãos responsáveis.

Os dados coletados são de grande importância epidemiológica, pois, além de identificar e analisar o perfil das tentativas de suicídio, em uma determinada região, revelam características importantes para o seu manejo e prevenção; isso auxilia a formulação de ações e políticas no âmbito da gestão em saúde, de modo a melhorar a qualidade de vida e reduzir os fatores de risco para tal agravo. Considerada a importância do tema, também, é necessário o desenvolvimento de projetos que visem à formação continuada dos profissionais de saúde, de forma que possam desenvolver competências e habilidades para garantir um atendimento de maior qualidade, a fim de atender às diferentes necessidades dos pacientes e, assim, efetivar uma assistência diferenciada.

A partir dos casos registrados, no período de estudo, foi possível perceber a incompletude de algumas informações dos pacientes atendidos pelo serviço, como escolaridade, profisssão e a raça/cor, mesmo com ausência, por vezes, do preenchimento destas variáveis, não influenciou a relevância do estudo.

Contudo, diante dos dados expostos e da magnitude desse tema, foi possível alcançar o objetivo deste estudo, embora seja necessário sugerir novas pesquisas, com outras variáveis, visto que, nesta verificação, foram caracterizados os casos que chegaram ao Centro de Informação e Assistência toxicológica do município de Cascavel. Quanto as limitações desse estudo, foram analisados os casos de tentativa de suicídio somente em um único centro entre os quatro centros localizados no Estado do Paraná. Contudo, também, é essencial, por parte dos profissionais de saúde, um acompanhamento cuidadoso quanto aos registros e quanto à assistência nos casos de tentativas de suicídio após o atendimento hospitalar.

É necessária uma assistência multidisciplinar, após a alta hospitalar em decorrência da tentativa de suicídio, com acompanhamento pela rede de serviços, para receber tratamento adequado de saúde mental e de fortalecimento das vulnerabilidades que possam conduzir esse sujeito a uma nova tentativa de suicídio.

\section{REFERÊNCIAS}

1. Organização Mundial da Saúde: Prevenção do suicídio: um recurso para conselheiros. [página na Internet]. Genebra/Suiça; 2006. [Atualizado 2020 maio 15]. Disponível em: https://www.who.int/mental_health/media/co unsellors_portuguese.pdf

2. Secretaria da Saúde. Boletim Vigilância epidemiológica de suicídio e tentativa de suicídio. Rio Grande do Sul. 2018;1(1): [online] [acesso em 2020 Mar. 20]. Disponível em:

https://www.cevs.rs.gov.br/upload/arquivos/2 01809/05162957-boletim-de-vigilanciaepidemiologica-de-suicidio-n1-2018.pdf. Acesso em: 20 mar. de 2020.

3. Botega NJ. Comportamento suicida: epidemiologia. Psicologia USP. São Paulo, 2014; 25(3)231-236, s/m. [online] [acesso em 2020 Abr. 07]. Disponível em: http://www.scielo.br/scielo.php?script=sci_art text\&pid=S0103-

$65642014000300231 \& \operatorname{lng}=\mathrm{en} \& \mathrm{nrm}=\mathrm{iso}$.

4. Bachmann S. Epidemiology of Suicide and the Psychiatric Perspective. International journal of environmental research and public health, s/l. 2018;15(7): s/p, [online] [acesso em 2020 Jul. 16]. Disponível em: https://pubmed.ncbi.nlm.nih.gov/29986446/.

5. Trigueiro A. Viver é a melhor opção: a prevenção do suicídio no Brasil e no mundo. São Bernardo do Campo: Correio Fraterno; 2015. 
6. Brasil. Ministério da Saúde. Secretaria de Vigilância em Saúde. Boletim Epidemiológico. Brasília, 2017;48(30). [página da internet] [Atualizado 2020 Mar. 20]. Disponível em: https://www.saude.gov.br/images/pdf/2017/se tembro/21/2017-025.

7. Silva TPS, Sougey EB, Silva J. Estigma social no comportamento suicida: reflexões bioéticas. Rev. Bioét., Brasília, 2015;23(2): 419-426, ago. [online] [acesso em 2020 Abr. 07]. Disponível em: http://www.scielo.br/scielo.php?script=sci_art text\&pid=S1983-

$80422015000200419 \& \operatorname{lng}=\mathrm{en} \& \mathrm{nrm}=$ iso.

8. World Health Organization. Relatório Mundial da Saúde. Saúde mental: nova concepção, nova esperança. Lisboa; 2002. [página da internet] [Atualizado 2020 Mar. 10]. Disponível em: https://www.who.int/whr/2001/en/whr01_djm essage_po.pdf?ua $=1$.

9. Ministério da Saúde (BR). Conselho Nacional de Saúde. Resolução 466/2012. Brasília; 2012.

10. Albuquerque PCC. Sistemas de informação em saúde e as intoxicações por agrotóxicos em Pernambuco. Revista brasileira de epidemiologia, São Paulo, 2015;18(3):666678, s/m. [online] [acesso em 2020 Jun. 04]. Disponível em: http://www.scielo.br/scielo.php?script=sci_art text\&pid=S1415790X2015000300666\&lng=en\&nrm=iso.

11. Dantas JS, Souza EL. Perfil do paciente com intoxicação exógena por chumbinho na abordagem inicial em serviço de emergência. Revista Eletrônica de Enfermagem, Ceará, 2013;15(1):54-60, [online] [acesso em 2020 Mar. 30]. Disponível em: https://revistas.ufg.br/fen/article/view/15506.

12. Moreira DL. Perfil de pacientes atendidos por tentativa de suicídio em um centro de assistência toxicológica. Revista Cienc. Enferm, 2015;21(2):63-75, ago. [online] [acesso em 2020 Maio 15]. Disponível em: https://scielo.conicyt.cl/pdf/cienf/v21n2/art_0 7.pdf.

13. Avanci RC. Perfil do adolescente que tenta suicídio em uma unidade de emergência. Revista Brasileira de Enfermagem, Brasília, 2005;58(5):535-539, Out. [online] [acesso em 2020 Mar. 30]. Disponível em: http://www.scielo.br/scielo.php?script=sci_art text\&pid=S0034-

$71672005000500007 \& \operatorname{lng}=\mathrm{en} \& \mathrm{nrm}=$ iso.

14. Veloso C. Violência autoinfligida por intoxicação exógena em um serviço de urgência e emergência. Revista Gaúcha Enfermagem, Porto Alegre. 2017;38(2):1-8. [online] [acesso em 2020 Mar. 02]. Disponível em:

http://www.scielo.br/scielo.php?script=sci_art text\&pid=S1983-

$14472017000200411 \& \operatorname{lng}=\mathrm{en} \& \mathrm{nrm}=$ iso.

15. Santos SA. Substâncias tóxicas e tentativas e suicídios: considerações sobre acesso e medidas restritivas. Cad. saúde colet., Rio de Janeiro. 2013;21(1):53-61. [online] [acesso em 2020 Jun. 25]. Disponível em: http://www.scielo.br/scielo.php?script=sci_art text\&pid=S1414-

$462 X 2013000100009 \& \operatorname{lng}=e n \& n r m=i s o$.

16. Gondim APS. Tentativas de suicídio por exposição a agentes tóxicos registradas em um Centro de Informação e Assistência Toxicológica em Fortaleza, Ceará, 2013. Epidemiol. Serv. Saúde, Brasília. 2017;26(1):109-11, mar. [online] [acesso em 2020 Abr. 07]. Disponível em: http://www.scielo.br/scielo.php?script=sci_art text\&pid=S2237$96222017000100109 \& \operatorname{lng}=\mathrm{en} \& \mathrm{nrm}=\mathrm{iso}$.

17. Queiroz CMS. Análise de casos de intoxicação por substâncias químicas e medicamentos no hospital geral do estado de alagoas. Infarma Ciências Farmacêuticas, 2013;23(5/6):46-60. [online] [acesso em 2020 Maio 04]. Disponível em: http://revistas.cff.org.br/?journal=infarma\&pa ge $=\operatorname{article} \& o p=v i e w \& p a t h \% 5 B \% 5 \mathrm{D}=378$.

Recebido em: 22.12.2020 Aprovado em: 27.06.2021 